\title{
Cardiac extrinsic compression due to abdominal distension as a cause of electrocardiographic abnormality in a critically ill patient
}

Juan Manuel Salvador-Casabón ${ }^{1, *}$, Javier Escota-Villanueva ${ }^{1}$, Paola Casanova-Esteban ${ }^{1}$, Ramón Ortiz-Giménez ${ }^{2}$, Daniel Cantero-Lozano ${ }^{1}$ and Juana María Pelegrín-Diaz ${ }^{1}$

\author{
${ }^{1}$ Cardiology department, Hospital Clínico Universitario "Lozano Blesa", Zaragoza, Spain \\ 2 Radiology department, Hospital Clínico Universitario "Lozano Blesa", Zaragoza, Spain \\ *Correspondence: juanma_cheick@hotmail.com (Juan Manuel Salvador-Casabón)
}

DOI: $10.31083 /$ i.jmcm.2020.03.826

This is an open access article under the CC BY 4.0 license (https://creativecommons.org/licenses/by/4.0/).

Anomalies in the ST segment, especially ST-segment elevation, require the ruling out of possible etiologies such as myocardial ischemia or pericarditis. However, other noncardiac disorders such as increases in intra-abdominal and intrathoracic pressure, may cause ST-segment elevation as well and should be considered as possible etiologies due to the poor prognosis they imply in the clinical evolution of the patient. This is especially important in critically ill patients. In this respect, we herein present a case in which the increase in intra-abdominal pressure and thoracic cavity elevation due to gastric and intestinal distension, which was not present in the first CT, provoked a cardiac and coronary compression resulting in T-shaped dome wave, a QT prolongation and ST segment elevation in the inferior leads of the electrocardiogram, the patient did not present at the time of admission, ruling out other possible etiologies such as ionic imbalance or acute ischemia.

\section{Keywords}

Abnormal electrocardiogram; ST segment elevation; QT prolongation; Spiked Helmet sign; abdominal distension

\section{Introduction}

The electrocardiogram is an important tool for diagnosing diseases that cause repolarization abnormalities, mainly ischemic heart disease. This is particularly important in those with ST elevation. However, in the critically ill patients, changes in repolarization (including ST elevation) have a low specificity due to the variety of non-ischemic processes that can alter the electrocardiogram and that must be taken into account [1].

Our present case illustrates that severe gastric and bowel distension secondary to paralytic ileus caused a displacement of the heart and compression of the posterior descending artery (PDA). This led to a T-shaped dome wave, QT prolongation and ST segment elevation in the inferior leads of the electrocardiogram that was not initially present.

\section{Case Report}

An 81-year-old male, with a past history of hypertension, paroxysmal atrial flutter and an episode of low digestive hemorrhage due to diverticulosis in April 2018.

He went to the emergency department in December 2018 due to a new episode of low digestive hemorrhage due to diverticulosis that caused hemodynamic instability (blood pressure 80/55 $\mathrm{mmHg}, \mathrm{Hb} 7.3 \mathrm{~g} / \mathrm{dL}$ ). An urgent abdominal computed tomography scan (CT) was performed revealing active bleeding in the descending colon. A surgical subtotal colectomy was performed with ileocolic mechanical anastomosis. Blood transfusions were also performed and the hemoglobin levels and blood pressure were corrected. After surgery, the patient presented good clinical evolution and was transferred to the floor unit. The electrocardiogram at that time showed sinus rhythm with narrow QRS without alterations in repolarization (Fig. 1)

On the $7^{\text {th }}$ day after the surgical intervention, he presented abdominal distension, hypotension and restlessness. Another urgent abdominal CT was performed which ruled out suture dehiscence but showed significant gastric and small bowel distension suggestive of postoperative paralytic ileus and elevation of the thoracic cavity secondarily. It also showed pleural effusion but no pericardial effusion. No signs of risk of acute intestinal ischemia like pneumatosis or intrahepatic portal vein gas were observed.

Absolute diet was prescribed and a nasogastric tube was placed. Due to dyspnea secondarily to distension (oxygen saturation of $87 \%$ ), the patient required non-invasive mechanical ventilation for a time.

Although the patient was without chest pain or other new symptomatology an electrocardiogram was performed. The electrocardiogram showed QT prolongation with a T-shaped dome wave with ST segment elevation in some QRS complexes of the inferior leads (Fig. 2). Blood sample showed: potassium $4.7 \mathrm{mEq} / \mathrm{l}$, magnesium $2.37 \mathrm{mg} / \mathrm{dL}$, total serum calcium $8.9 \mathrm{mg} / \mathrm{dL}$ and the rest ionic parameters were in normal range. In addition, the patient had no elevation of biomarkers of myocardial necrosis. The patient received antibiotic treatment with Ceftazimide, no antibiotics that could cause QT prolongation were used. In the following hours, 


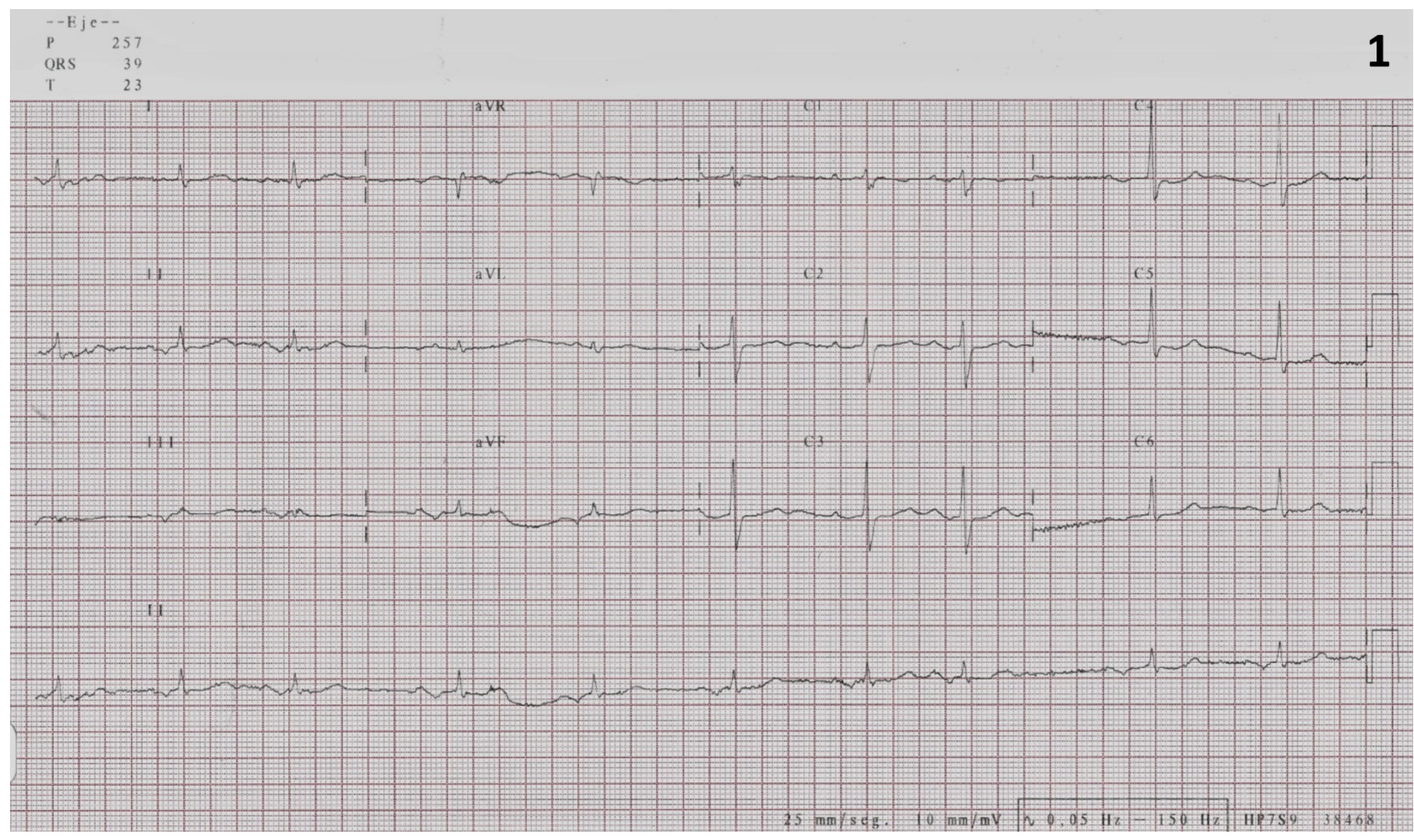

Fig. 1. Baseline electrocardiogram made at patient admission showing no repolarization abnormalities.

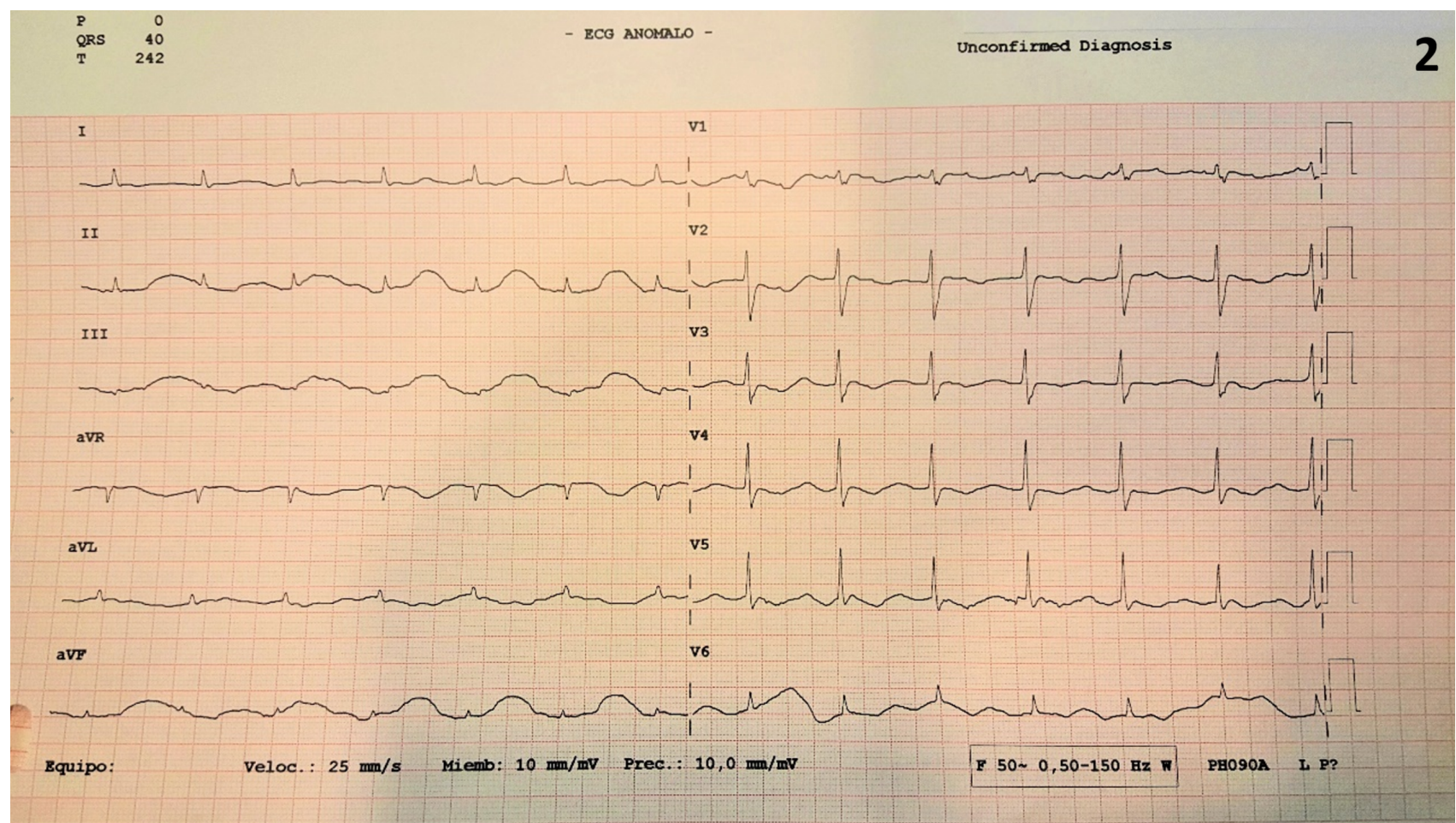

Fig. 2. Electrocardiogram when presenting the gastric distension showing QT prolongation and wide dome shaped T-wave with the ST segment elevation in some complexes of the inferior leads.

patient oxygenation improved and it was possible to remove the ventilatory support, while the electrocardiogram pattern was still present.

The CT was revalued and compared to the previous CT per- formed at the time of admission: it was observed that the bowel and gastric distension secondary to ileus not initially present, caused a compression of the inferior wall and posterior interventricular groove of the heart where the PDA and posterior interventricular 

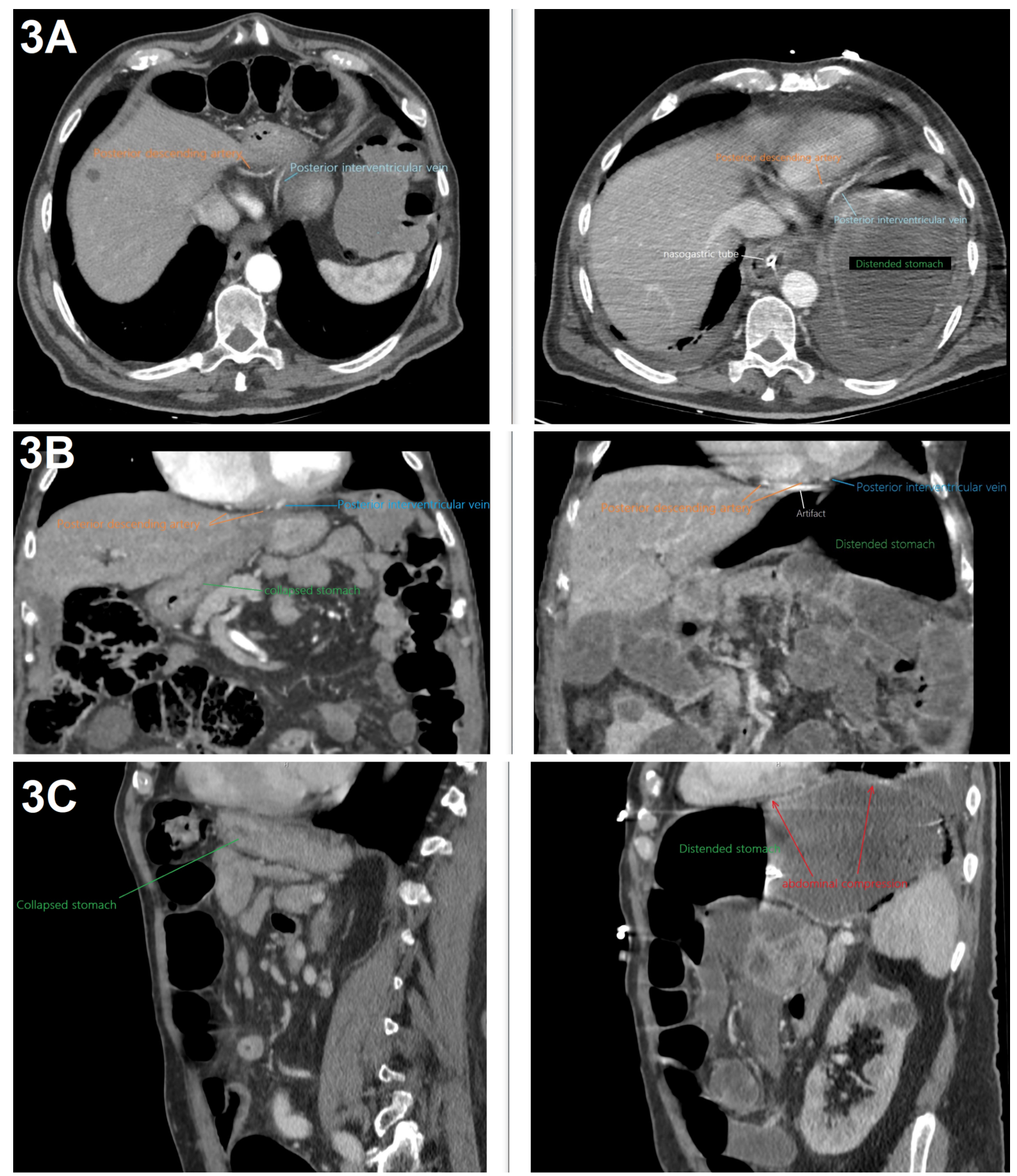

Fig. 3. CT scan thorax-abdominal at patient admission (left) and a week later (right). Severe bowel and stomach distension a week after admission (right images) not seen at patient admission (left) elevates the thoracic cavity and compresses posterior descending artery and posterior interventricular vein. A: Axial view. Right image is an upper level than left image due to abdominal distension. B: Coronal view. Posterior descending artery is observed in two sections. At the time of admission the stomach was collapsed, a week later significant distension was observed. C: Sagittal view. Thoracic cavity elevation due to abdominal distension is observed.

vein are located. (Fig. 3A-C). The PDA and the interventricular vein displayed similar contrast enhancement because the arterial phase of both CT were acquired to study abdominal vascularization.

Clinical evolution was unfavorable, as the patient showed no improvement in intrabdominal distension possibly triggering nonocclusive intestinal ischemia in the following hours, so conservative management and palliative sedation were performed and as a result, the patient eventually died in the following days.

\section{Discussion}

We present an infrequent case of electrocardiographic alteration of ST segment, QT prolongation and T-shaped dome wave, possibly due to external heart and PDA compression because of gastrointestinal distension, in a patient admitted to the intensive care unit for a cause unrelated to acute cardiological pathology.

This elevation of the thoracic cavity secondary to the gastrointestinal distension was not present in the initial CT (as can be seen comparing left and right Fig. 3A-C), so we hypothesized that changes in the electrocardiogram are secondary to that extrinsic 
compression on posterior epicardial vessels not initially present. This is the first case in which there is evidence of a patient with a normal electrocardiogram coinciding a CT with no distension and one week later the patient showed these electrocardiographic alterations coinciding with the presence of an elevation of the thoracic cavity and abdominal distension in the $\mathrm{CT}$. He reported neither chest nor acute epigastric pain (he felt bloating), in fact, the electrocardiogram was performed by chance and no CT signs of acute non-occlusive intestinal ischemia were present. Other etiologies that could cause similar repolarization alterations such as ionic imbalance or acute myocardial ischemia due to myocardial infarction, were ruled out.

This rare alteration in dome-shaped repolarization, resembles the "Spiked Helmet sign" when this alteration coincides with the QRS (because of its similarity to the military helmet of Prussia in the mid-nineteenth century). This has been previously described in some patients, mainly in those admitted to an intensive care unit [2]. Although the exact cause of the spiked helmet ST elevation is uncertain, the increase in both intrabdominal and intrathoracic pressure seem to be related to this cyclic electrocardiographic alteration $[2,3,4,5]$. This electrocardiographic pattern has been observed more frequently in limb leads (II, III, aVF). It has also been described in patients connected to mechanical ventilation with equally high intrathoracic pressures [4]. It has been postulated that the alterations in the inferior leads appear secondary to intra-abdominal pressure increases for different reasons while those with alterations in the precordial leads are secondary to increased intrathoracic pressure such as pneumothorax [4, 6].

This patient had an increase in both intrathoracic pressure due to mechanical ventilation and intra-abdominal pressure due to gastric distension and ileus but the electrocardiographic alterations were observed in the inferior leads. In addition, the CT showed an elevation of the thoracic cavity due to gastric distension and the pattern was still present once the ventilatory support was removed.

Our case resembles the one described by Singh et al. [7]. They postulated that increase in intra-abdominal pressure provoked external compression of the inferior epicardial arteries (right coronary and posterior descending arteries) with ST-segment elevation in the inferior leads but coronary arteries without obstructive lesions with resolution of the ST-elevation by decreasing intraabdominal pressure. As the PDA runs in the posterior interventricular groove the increase in intra-abdominal pressure affects it directly as the heart settles on the diaphragm, hence that could explain that increases in intra-abdominal pressure have impact in the inferior leads of the electrocardiogram and not in others. However, as many times there is no elevation of markers of myocardial necrosis, repetitive epidermal stretch related with the increase of pressure has been proposed as an underlying mechanism, suggesting that the clinical significance of this pattern is not to perform a cardiac catheterization, but focus on a possible critical abdominal event [6].Other suggested mechanisms are: stimulation of the diaphragm by the left phrenic nerve or direct stimulation of the inferior wall of the left ventricle by the diaphragm $[8,9]$

The presence of this or similar electrocardiographic pattern in the inferior leads has been described in some critically ill patients who present an increase in intra-abdominal pressure. The CT shows an elevation of the thoracic cavity that involves the inter- ventricular groove of the heart and the PDA, which was not present on the CT seven days before and that coincides with the changes in the electrocardiogram, so we hypothesized this may be the underlying mechanism of this alteration. Its early recognition as a marker of severity would contribute to a better characterization of the patient and when making therapeutic decisions since its presence seems to be associated with a poor short-term prognosis

\section{Ethics Approval and Consent to Participate}

This case report was conducted under the consent of the hospital and in accordance with the Declaration of Helsinki.

\section{Acknowledments}

We'd like to express our gratitude to editors and peer reviewers for their opinions and suggestions, which helped us during the writing of this manuscript.

\section{Conflict of interest}

The authors declare no conflicts of interest statement

Revised: August 26, 2020

Accepted: September 12, 2020

Published: September 20, 2020

\section{References}

[1] Rennyson SL, Hunt J, Haley MW, Norton HJ, Littmann L. Electrocardiographic ST-segment elevation myocardial infarction in critically ill patients: an observational cohort analysis. Crit Care Med. 2010; 38(12): 2304-2309.

[2] Littmann L, Monroe MH. The "spiked helmet" sign: a new electrocardiographic marker of critical illness and high risk of death. Mayo Clin Proc. 2011; 86(12): 1245-1246.

[3] Tomcsányi J, Frész T, Proctor P, Littmann L. Emergence and resolution of the electrocardiographic spiked helmet sign in acute noncardiac conditions. Am J Emerg Med. 2015; 33(1): e5-7.

[4] Littmann L, Proctor P. Real time recognition of the electrocardiographic "spiked helmet" sign in a critically ill patient with pneumothorax. Int J Cardiol. 2014; 173(3): e51-e52.

[5] Hibbs J, Orlandi Q, Olivari MT, Dickey W, Sharkey SW. Giant J Waves and ST-Segment Elevation Associated with Acute Gastric Distension. Circulation. 2016; 133(11): 1132-1134.

[6] Littmann L. Spiked helmet pattern ST elevation due to severe abdominal distension. J Electrocardiol. 2018;51(2):276-277.

[7] Singh M, Sood A, Rehman MU, Othman M, Afonso L. Elevated Hemi-diaphragms as a Cause of ST-segment Elevation: A case report and review of literature. J Electrocardiol. 2017; 50(5): 681-685.

[8] Brearley WD Jr, Taylor L 3rd, Haley MW, Littmann L. Pneumomediastinum mimicking acute ST-segment elevation myocardial infarction. Int J Cardiol. 2007; 117(2): e73-e75.

[9] Chase C, Brady WJ. Artifactual electrocardiographic change mimicking clinical abnormality on the ECG. Am J Emerg Med. 2000; 18(3): 312-316 